\title{
A QoS Control Scheme for Voice and Data Services in cdma2000 System
}

\author{
Omneya Issa and Jean-Charles Grégoire \\ INRS-EMT, Place Bonaventure, \\ 800, de la Gauchetière Ouest, bureau 6900 \\ Montréal (Québec), H5A 1K6 Canada \\ \{issa, gregoire\}@inrs-EMT.uquebec.ca
}

\begin{abstract}
The 3GPP2 standards gave many new features to cdma2000 to be able to support voice and data services, and established a layered structure for this network, but they left out QoS control as an open question. The aim of this paper is to present a QoS control scheme that exploits some of these new features. The proposed scheme consists of resolving contention between admitted services, scheduling the service packets and efficiently multiplexing data blocks on physical channels according to their QoS requirements. Its performance is evaluated using voice and data applications. It is compared with the performance of existing cdma2000 scheme in both indoor and outdoor environments.
\end{abstract}

\section{Introduction}

The new design of cdma2000 gives rise to a challenge concerning the use of these new capabilities and the control of QoS to get the maximum service satisfaction. Furthermore, the standards left out the QoS control as an open question. A QoS control scheme that exploits all these new features is needed. It must be able to resolve contention between service entities, schedule the service packets according to their QoS requirements and efficiently multiplex data blocks on physical channels. In fact, a form of priority scheduling is mentioned in the RLP standard [1]. That is, control messages have the highest priority followed by retransmitted data blocks, and, at the bottom, the data blocks that are being sent for the first time.

Some previous research efforts on cdma2000 [2] considered data burst scheduling between mobile users waiting to be admitted. However, the QoS control is left unspecified. [3] also proposed a framework for flow control in cdma2000. The framework deals with the bandwidth as a whole; it does not partition it into distinct traffic channels that differ in characteristics such as BER. Other researchers have proposed scheduling schemes for CDMA-based systems. But their work conforms rather to a hybrid TD/CDMA system, comparable to the forward packet data control function of 1XEV-DV extension of cdma2000 [1].

This paper proposes a QoS control scheme for the service connections that are admitted by the BS and hence the matching dedicated physical channels can be established. Section 2 describes the design components. Section 3 presents the simulation model and the design performance is investigated section 4 . 


\section{Proposed QoS Control Scheme}

The cdma2000 MAC layer contains a QoS control entity that is left unspecified. This section presents the design of a QoS control scheme that we propose for this undefined entity. This scheme controls the transmitted packets, both signalling and data packets, across their path through the layers based on requested QoS levels agreed to during service negotiation phase and the corresponding established physical channels. The QoS parameters of the service connections consist of the maximum delay (MaxD), the minimum throughput (MinTh) and the maximum loss [1].

Since the standards do not specify any particular classification, we choose to divide the traffic in four classes: control messages (CM) including messages such as IP routing, service signalling and handoff messages; bounded delay (BD) for traffic with a delay bound such as voice services; minimum throughput (MT) for traffic that requires minimum throughput such as ftp and http downloads; and best effort (BT) for the traffic that does not have any QoS requirements. Each class has its queue; when a connection is admitted, its packets are forwarded to their matching class queue.

It is mentioned in cdma2000 standards that priority levels can be used to provide over-the-air QoS, yet no specific priority assignment is defined. So we decide to assign a priority to each class shown in figure 1 and use a priority-based scheduler.

Our design deploys shaping of service traffic before being scheduled for helping the design to better commit to the agreed QoS and simplifying the design of the scheduler. The shaper of a data service with fixed-size packet simulates a CBR source by setting the bucket size B to one packet and token rate $r$ to the agreed service rate. A buffer is used to store packets that cannot pass because there is no waiting token. However, a voice service generates bursty traffic. A source model that matches the voice behavior is used by [4]. It assumes a two-state, on-off, Markov model; when on, voice user sends at the peak rate $\mathrm{P}$. The transition rate from on to off is $\alpha$, and the transition rate from off to on is $\lambda$. Based on this model, [4] deduced a relation (1) between $\mathrm{r}$ and $\mathrm{B}$ for $\mathrm{N}$ users in function of $\mathrm{P}, \alpha, \lambda$ and loss rate L. So we can determine $\mathrm{B}$ of a voice connection as a function of $\mathrm{r}$.

$$
[(\ln L) / N] r^{2}-[P \ln L+(\alpha+\lambda) B] r+\lambda N P B=0
$$

BE connections do not need shaping and the control messages pass without shaping, as well. Note that packets, that are released, pass through the RLP entity. The RLP entity fragments the packets into 266-bit PDUs (Protocol Data Unit), called data blocks (DBs), which are then forwarded to the class queue of their service connection.

Our priority-based scheduler is not sufficient to handle the different QoS needs of each class. So each class must have its own scheduling policy. Note that the overall scheduling scheme follows the standard RLP's. The CM queue has FIFO policy except that the handoff messages have higher priorities. An earliest deadline first policy is adopted in the BD queue; the packet deadline is the packet arrival time plus MaxD. When this queue is selected for service, all blocks whose deadlines have expired are dropped. A FIFO policy is adequate for MT queue since the throughput guarantee is handled by the admission phase. In this queue, if a block has the same arrival time as a RLP retransmitted block, the retransmitted one will be scheduled first. The $\mathrm{DBs}$ of $\mathrm{BE}$ queue are sorted according to their connection priority (if specified). 


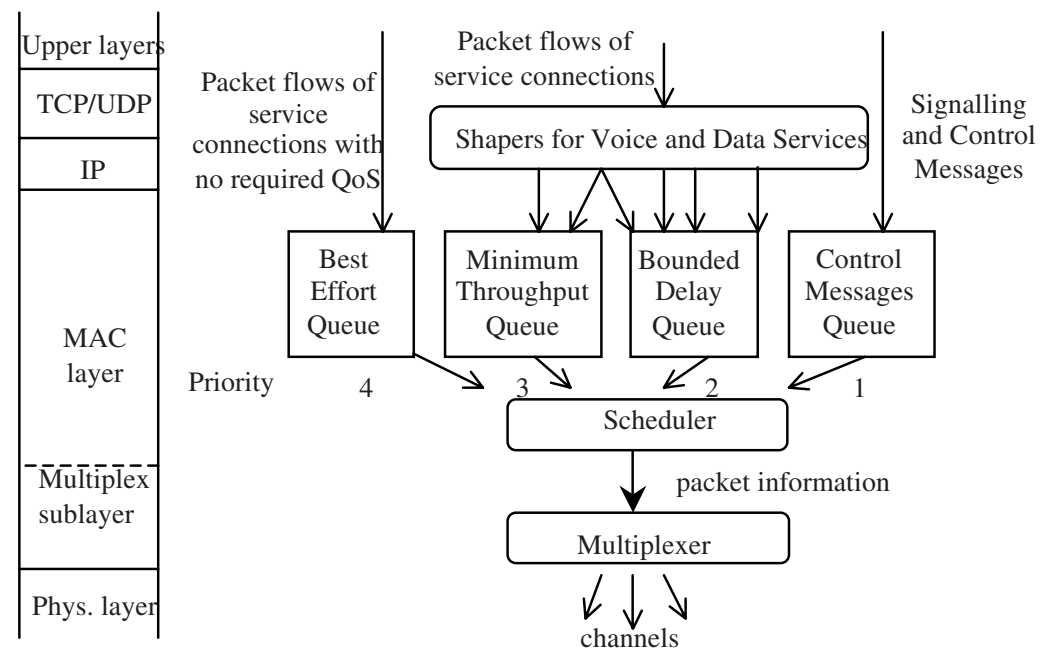

Fig. 1. QoS Control Scheme.

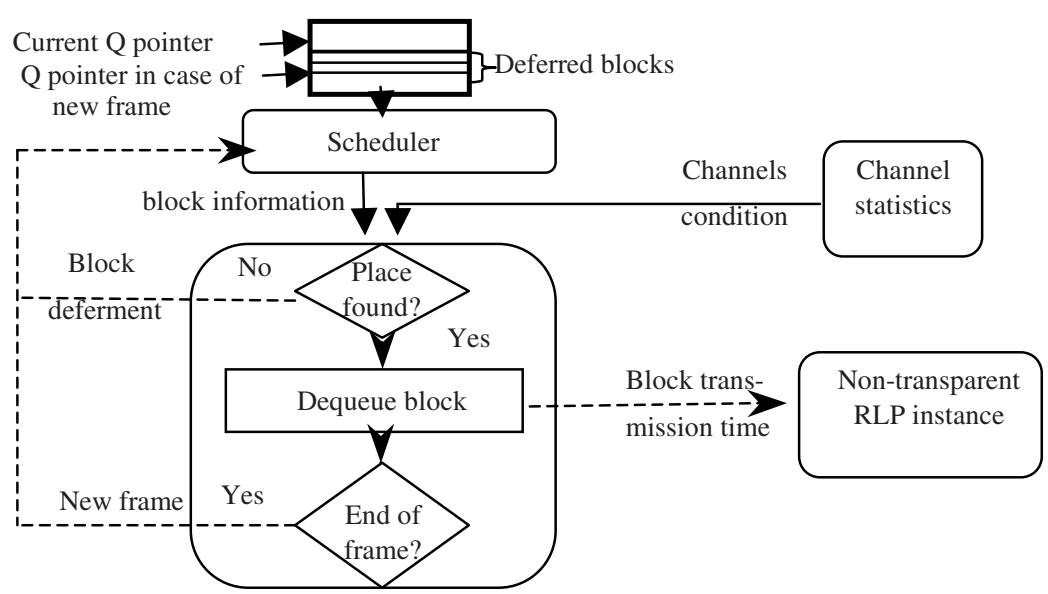

Fig. 2. The multiplexer.

The multiplexer is responsible for multiplexing DBs onto the adequate channels. The standards only specify a multiplex option that defines the maximum number of DBs and the allowed block size for a channel. They do not specify the way a channel is selected for DB transmission. In our design, figure 2, the multiplexer first checks the presence of a place on a channel supporting the BER required by the DB connection or a lower BER. A place on a channel is not found when the channel is reported in error by the statistics gathering done at this sublayer. The DB transmission time is reported to the RLP to avoid a useless generation of a duplicate DB in case of the reception of an early duplicate NAK for this DB. When the multiplexer fails to transmit a DB, the latter is not discarded; it is left at the head of the queue. When the multiplexer signals a new frame, the scheduler begins with the DBs that were deferred 
within the last frame and are still at the head of the served queue. This compensates for the DBs that should have been sent in the previous frame.

\section{Simulation Model}

The proposed scheme is implemented using the ns- 2 over both the MS and the BS nodes. We simulate a MS connected to a BS in a cmda2000 network and communicating with a remote host on a fixed network. The propagation delay is $3 \mu \mathrm{s}$ on the wireless network, and $100 \mathrm{~ms}$ on the fixed network. The voice is modelled as in [4], $\alpha$ is set to $1 / 1.2 \mathrm{~s}, \lambda$ to $1 / 1.8 \mathrm{~s}, \mathrm{~L}$ to $10^{-3}, \mathrm{P}$ to $26.6 \mathrm{kbps}, \mathrm{r}$ to $13.3 \mathrm{kbps}, \mathrm{B}$ to 180 packets and packet size is 50 bytes. The FTP application from ns- 2 is used, packet size is 512 bytes. The web browsing traffic is modelled as in [5]. Simulation models for both the cdma2000 forward and reverse link are created according to the standards. Slow fading is modelled by lognormal shadow fading, table 1. Fast fading is modelled by the superposition of 2 Rayleigh-distributed faded paths. Additive white Gaussian noise is added to simulate the effect of overall interference. The radio configuration of reverse and forward channels is 4 and 5 respectively. The BER of the fundamental $(\mathrm{FCH})$ and supplemental channel $(\mathrm{SCH})$ is $10^{-3}$ and $10^{-5}$ respectively. The frame length is $20 \mathrm{~ms}$.

Table 1. Parameters of the two simulated environments.

\begin{tabular}{|l|c|c|}
\hline & Indoor Environment & Outdoor Environment \\
\hline Model & In building & Vehicular \\
\hline Place & Office (soft partition) & Shadowed urban area \\
\hline Velocity & $3 \mathrm{~km} / \mathrm{h}$ & $100 \mathrm{~km} / \mathrm{h}$ \\
\hline Path loss exponent & 1.6 & 3 \\
\hline Shadowing deviation & 9.6 & 4 \\
\hline
\end{tabular}

\section{Simulation Results}

The simulation incorporates a FTP upload generated by the MS, 3 web sessions and a voice conversation. The MaxD of the voice is $100 \mathrm{~ms}$, the MinTh of FTP varies from $26.6 \mathrm{kbps}$ to $113.7 \mathrm{kbps}$. Note that the effective channel rate is lower than the nominal data rate of the standards because of the presence of headers in the transmitted frame. FTP and web sessions use non-transparent RLP while voice has a transparent one. The satisfaction of each application is measured according to its most pertinent QoS parameter. For FTP, the throughput is measured (Tm). For the voice, the percentage of discarded DBs (Pdp) due to expiration is presented. For web sessions, the average response time (RT) experienced by web pages is measured, that is, the time between the launching of the web page and the arrival of the entire page objects.

To investigate the performance of the proposed scheduler, without the effect of multiplexer, only one reverse channel is established with a rate that varies from $28.8 \mathrm{kbps}$ and $115.2 \mathrm{kbps}$, done by varying the transmission power and the target SIR. The DBs of the 3 applications are transmitted on this channel. A forward $57.6 \mathrm{kbps}$ 
SCH is opened for TCP ACKs and web sessions replies. Figure 3(a,b) shows that the proposed scheduler raises the Tm of FTP in indoor environments with a difference of $1-8 \mathrm{kbps}$ comparing to the scheduling of RLP standard. In outdoor environments, Tm is lower (2-6kbps) because the channels experienced more successive frame errors.

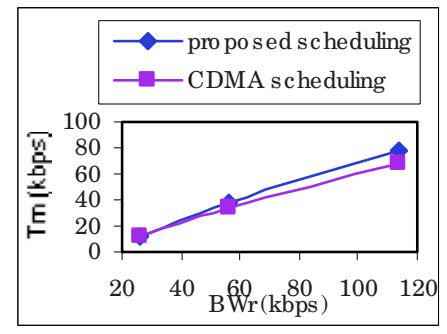

(a) FTP in indoor environment

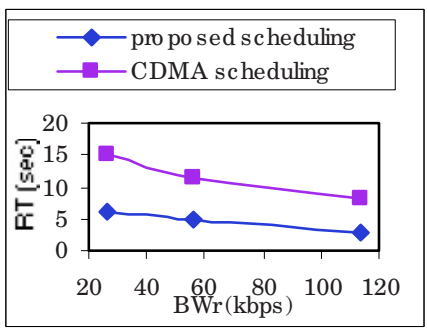

(c)Web in indoor environment

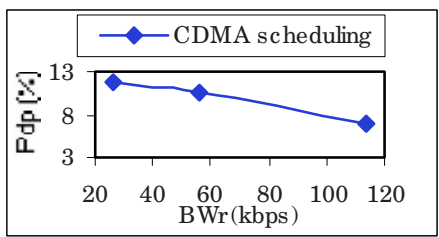

(e) Voice in indoor environment

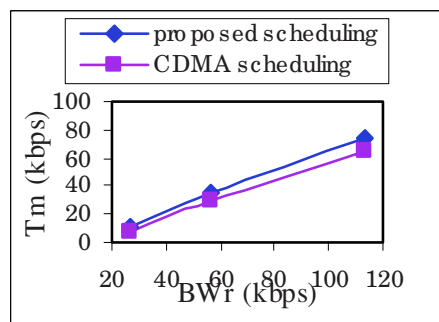

(b) FTP in outdoor environment

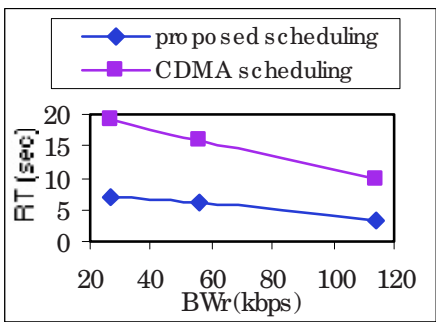

(d) Web in outdoor environment

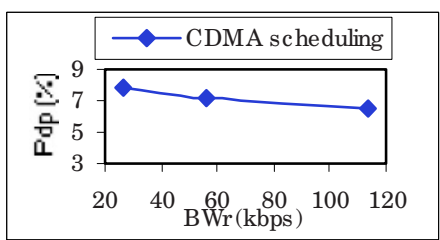

(f) Voice in outdoor environment

Fig. 3. Scheduler performance.

The difference in web RT using the proposed scheduler vs. RLP scheduling is 9s that goes down to $5 \mathrm{~s}$ when doubling the reverse bandwidth $(\mathrm{BWr})$ in indoor environment. In outdoor environment, this difference goes up to $12 \mathrm{~s}$ and decreases to $7 \mathrm{~s}$ when doubling $\mathrm{BWr}$, figure 3(c,d). When deploying our proposed scheduler, no voice packet is discarded because those packets have the priority of transmission. Whereas, in cdma2000 scheduling case, the new packets are served on a FIFO-basis, so voice blocks may wait till expiration. In indoor environments, Pdp is $11.5-7 \%$. In outdoor environments, Pdp goes from $8 \%$ to $6.5 \%$ because FTP packet generation rate is lower, due to successive errors, figure $3(\mathrm{e}, \mathrm{f})$. Note that the discarded voice blocks remain in queue for longer time which results in web request packets and FTP packets waiting longer before being transmitted, which increases the TCP estimation of round-trip time (RTT) and thus, reduces FTP Tm and increases web RT. 
Due to channel errors and its effect on TCP connections, Tm does not reach the required one, although the channel bandwidth is adequate to hold the required rate. That is, some frames are transmitted empty. When transmitting a voice conversation on a $14.4 \mathrm{kbps}$ channel, adequate for the average voice rate $13.3 \mathrm{kbps}, 20 \%$ of the frames are empty; those frames should carry DBs discarded due to expiration. Those idle frames can be saved by using the cdma2000 variable channel rate capability.

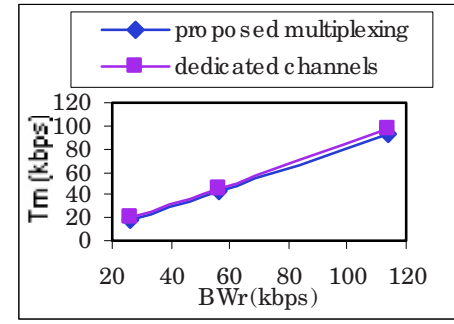

(a) FTP in indoor environment

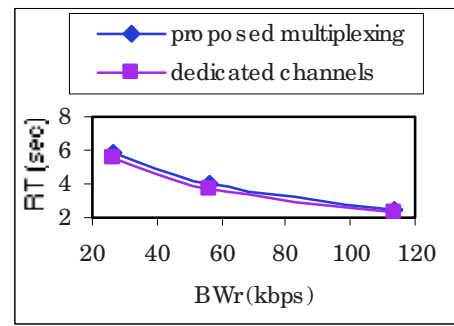

(c)Web in indoor environment

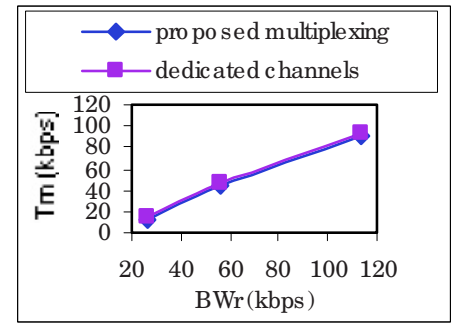

(b) FTP in outdoor environment

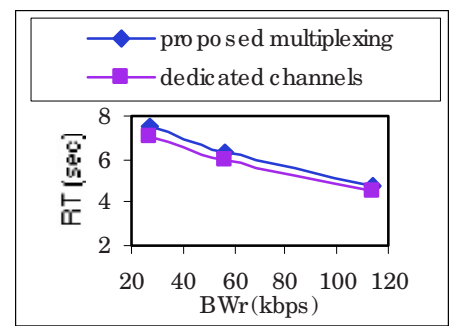

(d) Web in outdoor environment

Fig. 4. Multiplexer performance.

Another solution would be to give other services the possibility to transmit their DBs on the same channel, and thus, avoid opening new dedicated channels for those services. This solution favours the proposed multiplexing scheme. Figure 4 shows the results of simulating a FTP service and 3 web sessions that require a BER of $10^{-5}$ whereas the voice service needs a $10^{-3}$ BER. 2 reverse channels are opened, one $14.4 \mathrm{kbps} \mathrm{FCH}$ channel and one $\mathrm{SCH}$ channel whose rate varies from $28.8 \mathrm{kbps}$ to $115.2 \mathrm{kbps}$. A forward 57.6kbps SCH is opened as well. The BER of the FCH only fits the voice service, while the BER of SCH fits the 3 applications. To measure the effect of multiplexing on the applications' performance, we compare it with their performance when opening a dedicated channel adequate for each. When compared with the throughput of dedicated channel, the Tm of FTP using the proposed multiplexing is only affected by a difference of $0.5 \mathrm{kbps}$ in low rates that goes up to $2 \mathrm{kbps}$ in $113.7 \mathrm{kbps}$ rate. The web RT rose by $0.4 \mathrm{~s}$ in low $\mathrm{BWr}$ and by $0.2 \mathrm{~s}$ when reaching a $\mathrm{BWr}$ of $115.2 \mathrm{kbps}$. The voice Pdp is about $20 \%$ in the case of dedicated channel while no packet is discarded when using the proposed scheme. Those differences are comparatively the same in both indoor and outdoor environments. This shows that we can avoid the opening new dedicated channels for applications like web sessions, email and telnet and the expiration of voice packets by multiplexing the DBs of several services on the same channel when it fulfills their QoS requirements. 


\section{References}

1. Cdma2000 standards at http://www.3gpp2.org/Public_html/specs/index.cfm.

2. Nanda et al. Adaptation techniques in wireless packet data services. IEEE Comm.Mag. 2000.

3. Comaniciu, N. et al. QoS Guarantees for 3G CDMA Systems via Admission and Flow Control. VTC'2000, Boston, MA, September 2000.

4. J. Rosenberg. Engineering VBR Traffic Parameters for WAN Voice. (ICC '97), Canada.

5. The cdma2000 ITU-R Candidate Submission. April 1998. 